\title{
Glucocorticoid receptor polymorphisms do not affect the therapy efficiency in adult, Hungarian patients with 21- hydroxylase deficiency
}

\author{
Klára Koncz ${ }^{1,2}$, Abigél Farkas ${ }^{1}$, Marton Doleschall ${ }^{3}$, Nikolette Szucs ${ }^{1}$, Miklos Toth ${ }^{1}$, Peter Igaz ${ }^{1}$, Karoly Racz ${ }^{1}$, Attila Patocs $^{1,2}$ \\ ${ }^{1} 2^{\text {nd }}$ Department of Medicine, Semmelweis University, Budapest, Hungary \\ 2 "Lendület" Hereditary Endocrine Tumors Research Group, Hungarian Academy of Sciences - Semmelweis University, Budapest, Hungary \\ ${ }^{3}$ Molecular Medicine Research Group, Hungarian Academy of Sciences - Semmelweis University, Budapest, Hungary
}

\section{Background}

Congenital adrenal hyperplasia $(\mathrm{CAH})$ is an autosomal recessive inherited disorder caused by 21 -hydroxylase deficiency in $95 \%$ of all cases. Two main clinical subtypes: the classical (manifested after birth or in early newborn period) and the late onset (LO) phenotype (manifested commonly during puberty) can be distinguished. The lifelong glucocorticoid (GC) supplementation is essential in therapy of these patients. Response and need for GC therapy is individual and partly genetically determined.
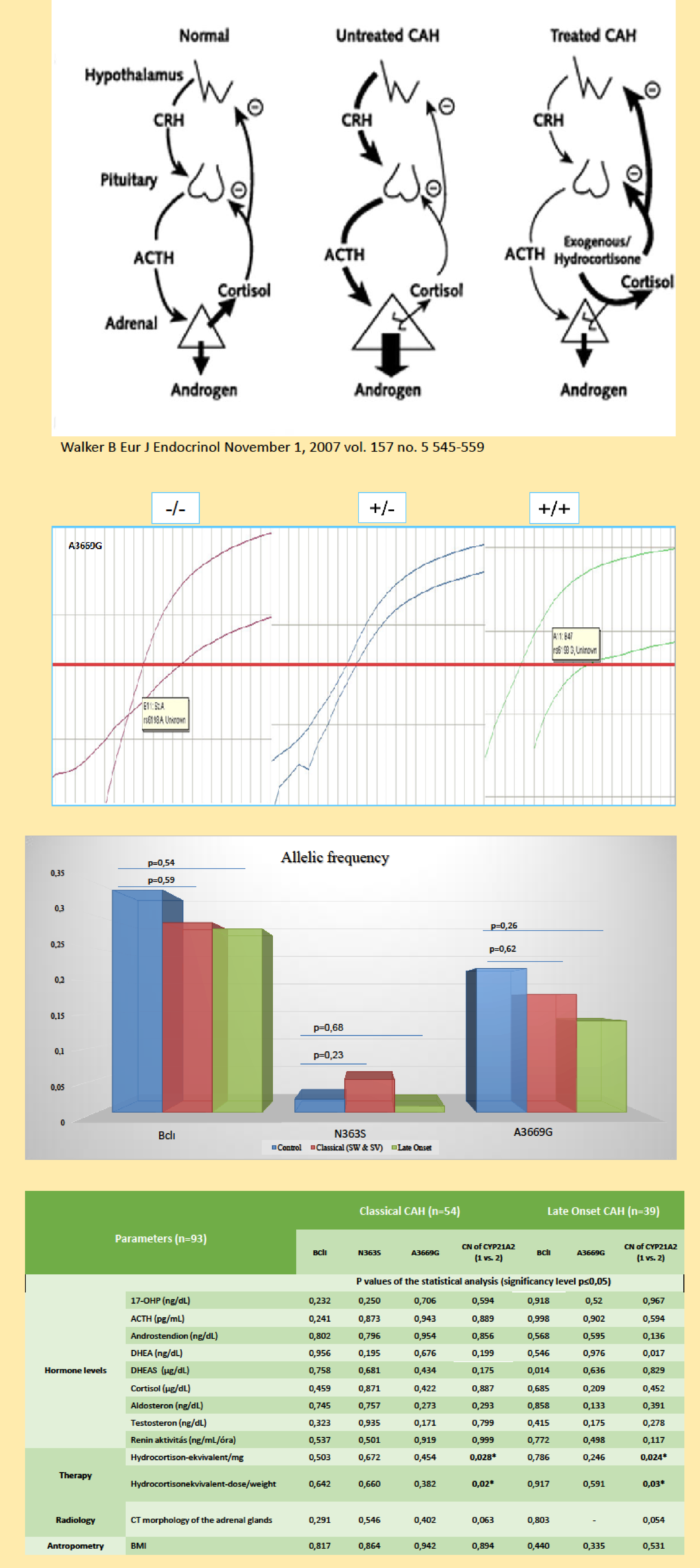

\section{Objective}

- Our aim was to study the effect of three well-known polymorphisms (SNPs) (BclI, N363S, A3669G) of the GR gene on therapy, clinical and laboratory parameters in adult Hungarian patients with 21hydroxylase deficiency.

- To examine whether the copy number $(\mathrm{CN})$ of the CYP21A2 gene influences the therapy in our patients.

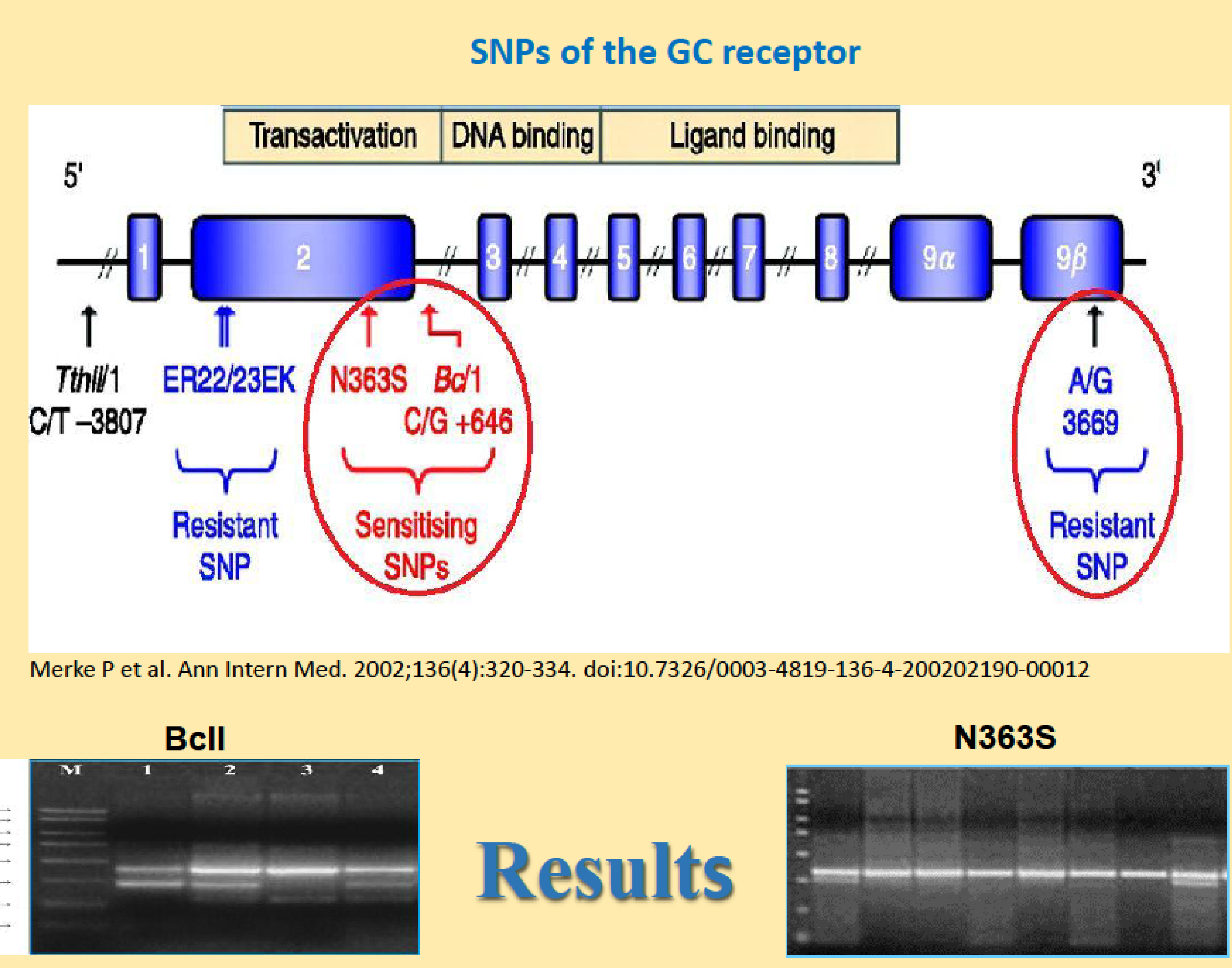

Allelic frequency of the GR polymorphisms did not differ from those observed in control population and did not associate with clinical, laboratory parameters or dosage of the GC supplementation. The copy number of the CYP21A2 in both groups of patients significantly negatively correlated with GC therapy $(\mathbf{p}=\mathbf{0 , 0 3})$.

\section{Discussion}

The GR polymorphisms-related sensitivity against glucocorticoids is not a major factor in determination of the dose of glucocorticoid supplementation in Hungarian, adult patients with $\mathrm{CAH}$. The CYP21A2 copy number measurement is a rapid, cheap and sensitive method for prediction the GC need in patients with 21-hydroxylase deficiency.

\section{Patients and Methods}

The diagnosis of 21-hydroxylase deficiency was based on clinical, laboratory and molecular genetic tests including the identification of the most common CYP21A2 gene mutations and determination of the allelic copy number $(\mathrm{CN})$ of the CYP21A2 gene.

In 93 patients (54 classical: 34 salt wasting +20 simple virilising and 39 late-onset, age: 28,8 13,7 years) with 21-hydroxylase deficiency the $\mathrm{BclI}$ and N363S polymorphisms were measured using allele-specific PCR, the A3669G polymorphism and the $\mathrm{CN}$ variations were detected by real-time qPCR. Allele frequencies of GR polymorphisms were compared to a Hungarian, control population $(\mathrm{n}=160)$. Association between GR SNPs and clinical, hormone laboratory and GC supplementation dosage was studied. For statistical analysis were used ANOVA, Student $\mathrm{t}$ test, Fisher's exact test and Chi-square test.

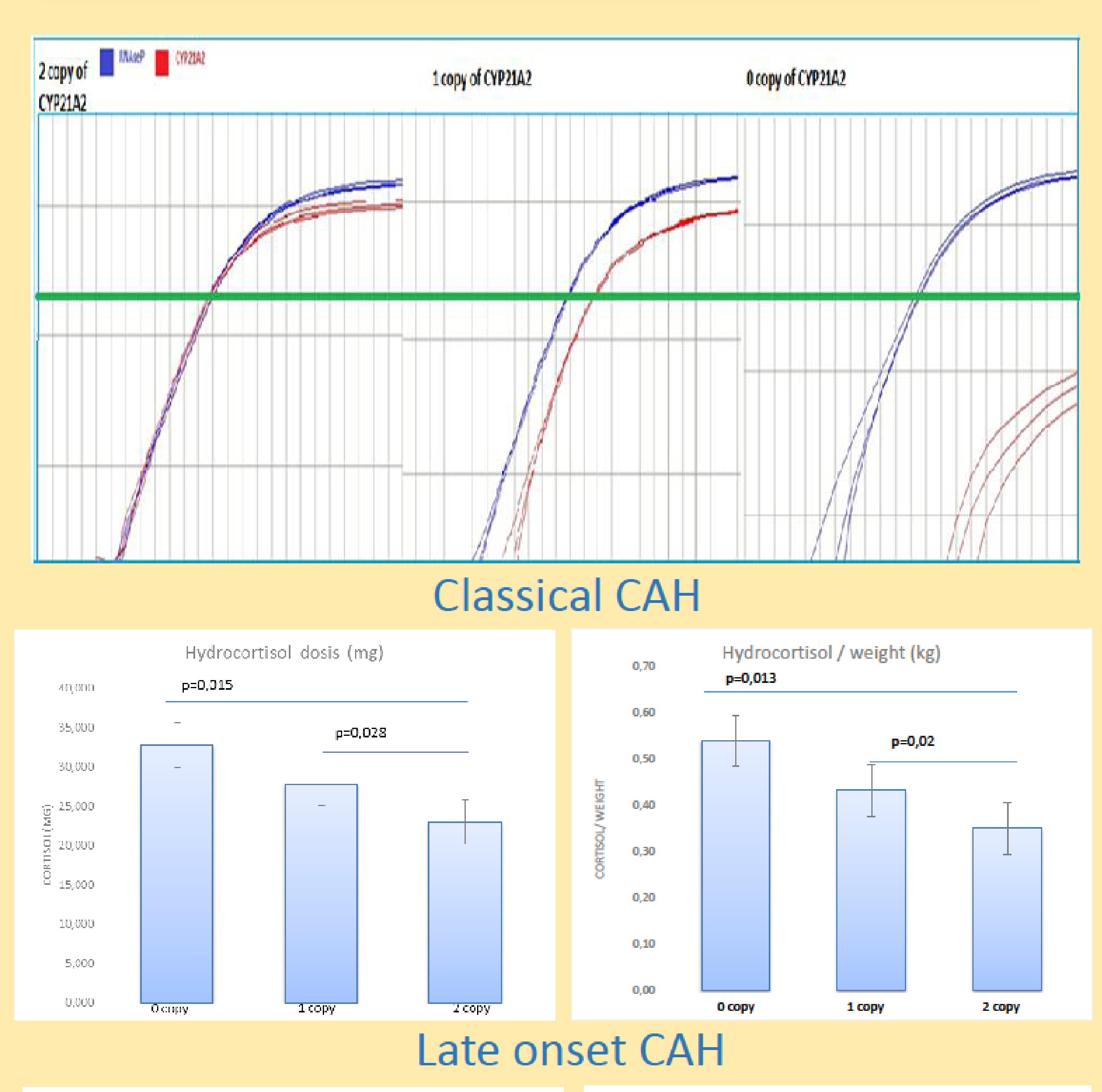

The authors acknowledge the financial support from Hungarian Research Found (OTKA PD100648) and from National Development Agency (KTIA_AIK-2-2012-0010) 